
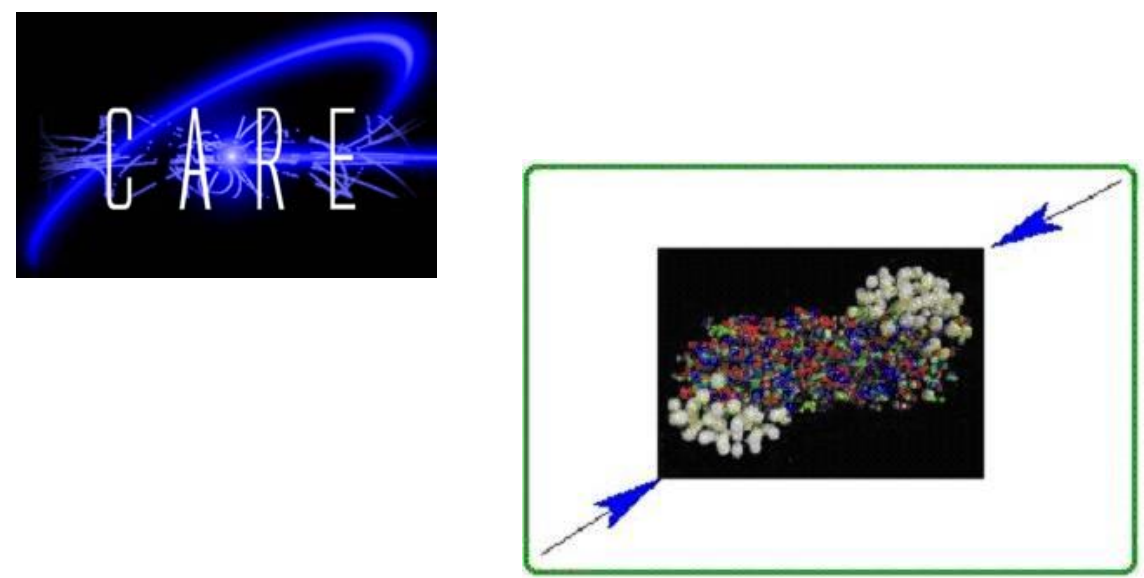

High Energy

High Intensity

Hadron Beams

\title{
Beam-Beam Effect with an External Noise in LHC
}

\author{
K. Ohmi ${ }^{1}$, R. Calaga ${ }^{2}$, W. Hofle ${ }^{3}$, R. Thomas ${ }^{3}$, F. Zimmermann ${ }^{3}$ \\ 1) KEK, Tsukuba, Japan \\ 2) BNL, Upton, NY, USA \\ 3) CERN, Geneva, Switzerland
}

\begin{abstract}
In absence of synchrotron radiation, proton beams do not have any damping mechanism for incoherent betatron motion. A noise, which kicks beam particles in the transverse plane, gives a coherent betatron amplitude. If the system is linear, the coherent motion is maintained in amplitude. Nonlinear force, beam-beam and beam-electron cloud interactions, cause a decoherence of the betatron motion keeping the amplitude of each beam particle, with the result that an emittance growth arises. We focus only on fast noise with a correlation time of 1-100 turns. Slower noise is less serious, because it is regarded as an adiabatic change like a closed orbit change. As sources of the noise, we consider the bunch by bunch feedback system and phase jitter of cavities which turns to transverse noise via a crab cavity.
\end{abstract}

Contribution to the " $22^{\text {nd }}$ Particle Accelerator Conference PAC07", Albuquerque (USA), 25-29 June 2007

Work supported by the European Community-Research Infrastructure Activity under the FP6 "Structuring the European Research Area" programme (CARE, contract number RII3-CT2003-506395) 


\title{
BEAM-BEAM EFFECT WITH AN EXTERNL NOISE IN LHC
}

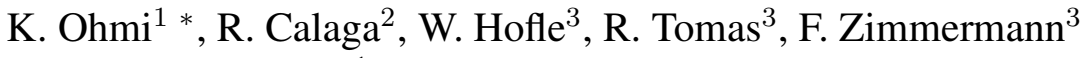 \\ ${ }^{1} \mathrm{KEK}$, Tsukuba, Japan \\ ${ }^{2}$ BNL, Upton, NY ${ }^{3}$ CERN, Geneva, Switzerland
}

\section{Abstract}

In absence of synchrotron radiation, proton beams do not have any damping mechanism for incoherent betatron motion. A noise, which kicks beam particles in the transverse plane, gives a coherent betatron amplitude. If the system is linear, the coherent motion is maintained in amplitude. Nonlinear force, beam-beam and beam-electron cloud interactions, cause a decoherence of the betatron motion keeping the amplitude of each beam particle, with the result that an emittance growth arises. We focus only on fast noise with a correlation time of 1-100 turns. Slower noise is less serious, because it is regarded as an adiabatic change like a closed orbit change. As sources of the noise, we consider the bunch by bunch feedback system and phase jitter of cavities which turns to transverse noise via a crab cavity.

\section{INTRODUCTION}

An external noise, which kicks beam transversely, induces an offset on the beam-beam collision. When the centroids between two colliding beams deviate by $\delta x$ at the collision point, the luminosity degrades geometrically as

$$
L(\delta x)=L_{0} \exp \left(-\frac{\delta x^{2}}{2 \sigma_{x}^{2}}\right)
$$

where $L_{0}$ and $\sigma_{x}$ are the luminosity and the beam size without deviation. When $\delta x$ fluctuates with an rms value $\left\langle\delta x^{2}\right\rangle$, the averaged luminosity is given by

$$
\langle L\rangle=L_{0}\left(1-\frac{\left\langle\delta x^{2}\right\rangle}{2 \sigma_{x}^{2}}\right)
$$

The degradation is negligible for $\delta x / \sigma \ll 1$.

As commonly known, the beam-beam interaction is strongly nonlinear. The collision offset caused by the noise lead to a diffusion of the particle motion[1], and induces a coherent oscillation between the two beams. The coherent motion is transferred to emittance growth due to its smearout[2]. We treat an emittance growth and luminosity degradation induced by an external noise in the beam-beam interaction. The emittance growth is analyzed by the weakstrong and strong-strong models, in which beam particles move in a potential given by the colliding beam as a fixed charged distribution, and the two beams move with interacting on each other.

Parameters of LHC are listed in Table 1.
Table 1: Basic parameters of LHC

\begin{tabular}{|l|c|cc|}
\hline variable & symbol & nominal & upgrade \\
\hline circumference & $L$ & $26,658 \mathrm{~m}$ \\
beam energy & $E$ & \multicolumn{2}{|c|}{$7 \mathrm{TeV}$} \\
bunch population & $N_{b}$ & $1.15 \times 10^{11}$ & $1.7 \times 10^{11}$ \\
half crossing angle & $\theta$ & $0.14 \mathrm{mrad}$ & $0.22 \mathrm{mrad}$ \\
beta function at IP & $\beta_{x, y}^{*}$ & $0.55 \mathrm{~m}$ & $0.25 \mathrm{~m}$ \\
emittance & $\varepsilon_{r}$ & $5.07 \times 10^{-10} \mathrm{~m}$ \\
beam-beam tune shift & $\xi$ & 0.0033 \\
bunch length & $\sigma_{z}$ & $7 \mathrm{~cm}$ & $3.78 \mathrm{~cm}$ \\
synchrotron tune & $\nu_{s}$ & 0.0019 \\
betatron tune & $\nu_{x(y)}$ & $63.31 / 59.32$ \\
revolution frequency & $f_{0}$ & $10^{9} / \mathrm{day}$ \\
\hline
\end{tabular}

\section{NOISE SOURCES}

The crab cavity can be a source of diffusion. Since the crab cavity is operated by a transverse mode, the deviation and jitter of RF phase give a dipole kick to the beam, with the result that a transverse offset at the collision point is generated. Both phases, the main RF and crab cavity RF, can be a source of the transverse offset.

Jitters of RF phase of the main cavity cause a deviation of timing of beam arrival at the crab cavity. The transverse offset, which arises from the jitter of the main RF system, is expressed by

$$
\delta x=\frac{c \tan \theta}{\omega_{R F}} \delta \phi_{R F} .
$$

where $\delta \phi_{R F}$ is the phase error of the main RF system.

The crab cavity gives a transverse kick due to its jitters of RF phase, with the result that the offset given by the kick is expressed as follows,

$$
\delta x=\frac{c \tan \phi}{\omega_{R F}} \frac{\cos \left[\pi \nu_{x}-\Delta \Phi\left(s^{*}, s_{c}\right)\right]}{2 \sin \pi \nu_{x}} \delta \phi_{c r a b},
$$

where $\Delta \Phi\left(s^{*}, s_{c}\right)$ and $\delta \phi_{R F}$ are the betatron phase difference between the collision point and the crab cavity and the deviation of the RF phase of the crab cavity, respectively. In the both cases, the jitter of the transverse offset is given by $\delta x \approx c \tan \phi \delta \psi / \omega_{R F}$.

Transverse bunch by bunch feedback systems damps the transverse dipole motion of the beam. Errors of the position monitor signal and kicker noise give a transverse kick 
on the beam. Betatron amplitude is transferred by the feedback system as follows

$$
\begin{aligned}
X_{i+1} & =X_{i}-G\left(\left\langle X_{i}\right\rangle+\delta X_{m o n}\right)+\delta X_{k i c k} \\
& =X_{i}-G\left(\left\langle X_{i}\right\rangle+\delta X_{m o n}\right)+\delta X_{k i c k}
\end{aligned}
$$

where $G, \delta X_{k i c k}$ and $\delta X_{m o n}$ are the feedback damping rate, kicker noise and monitor noise, respectively. The fluctuation of the betatron amplitude is given by

$$
\left\langle X^{2}\right\rangle=\frac{1}{2 G}\left(G^{2}\left\langle X_{m o n}^{2}\right\rangle+\left\langle X_{k i c k}^{2}\right\rangle\right)
$$

These fluctuations induce a diffusion in betatron amplitude and an excitation of a coherent beam-beam mode.

Two type of implementation for noise are installed in the simulation codes. First type of noise is expressed by a transformation

$$
T_{1}(-\delta) \exp \left(-U_{c o l}\right) T_{1}(\delta) M_{0}
$$

where

$$
T_{1}(\delta)\left(\begin{array}{c}
x \\
y
\end{array}\right)=\left(\begin{array}{l}
x+\delta_{x} \\
y+\delta_{y}
\end{array}\right) .
$$

$\delta$ 's are random variables, which are common values for every macro-particles. RF feedback system of accelerating and crab cavities is closed and is little influenced by the beam-beam interaction. We use this transformation for studying the crab cavity type of noise.

Bunch by bunch feedback systems kick the beam to reduce its coherent betatron amplitude. Beam-beam interaction can influence the feedback system. The transformation in the case of the bunch by bunch feedback system is characterized by two variables, damping rate and fluctuation, as follows,

$$
\exp \left(-U_{c o l}\right) T_{2}(\delta, \tau) M_{0}
$$

where

$$
T_{2}(\delta, G)\left(\begin{array}{c}
x \\
y
\end{array}\right)=\left(\begin{array}{c}
x-G\langle x\rangle+\delta_{x} \\
y-G\langle y\rangle+\delta_{y}
\end{array}\right) .
$$

Actually there is no difference between the two types of noises. The crab cavity also is equipped with a feedback loop to stabilize the phase.

\section{SIMULATION OF BEAM-BEAM INTERACTIONS WITH THE NOISES}

We execute a weak-strong and strong-strong simulation to study the noise effects. The strong-strong simulation contains a numerical noise due to the statistics of macroparticles. Indeed the dipole moment fluctuates $\sigma / \sqrt{N}$ turn by turn. The simulation gives an artificial emittance growth due to the numerical noise; the external noise less than the numerical noise is not visible. The number of particles should be increased according to the noise level and emittance growth rate to be studied.

\section{Crab cavity type of noise}

We first discuss the beam-beam effect with the noise given by Eq.(8). Figure 1 shows the evolution of emittance and luminosity for various noise amplitudes given by the weak-strong simulation. The emittance growth rate and luminosity decrement, which are estimated are shown in Figure 1. In the weak-strong simulation, macro-particles move in a static potential. Nonlinear beam-beam force (potential) can cause emittance growth even in absence of noise. Since the beam-beam tune shift is rather small $(\xi=0.0033)$, the motion is near solvable, therefore emittance growth is very weak, $<10^{-10}$. For increasing noise amplitude, the emittance growth and luminosity decrement become visible in the simulation. Since the revolution frequency is $\sim 10^{9}$ per day, the luminosity decrement $10^{-9}$ corresponds to 1 day life time. A noise level $\delta x=0.1-0.2 \%$ is the limit for 1 day luminosity life time.
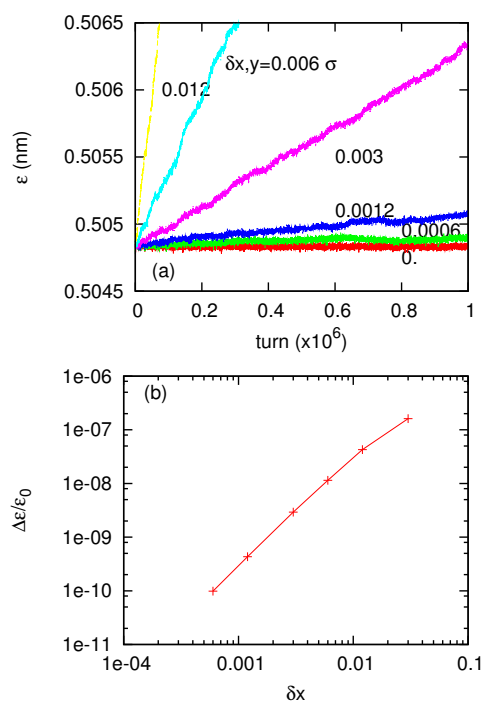

Figure 1: Emittance growth due to noise given by a weakstrong simulation. Plots (a) and (b) depict the evolution of emittance for various noise amplitude and their emittance growth rate, respectively.

In the weak-strong simulation, the noise does not induce a coherent beam-beam mode. The effect due to excitation of a coherent motion is estimated by the strong-strong simulation. The simulations were done with 1,000,000 macroparticles. The simulation contains an intrinsic error due to the statistics of the number of macro-particles [3]. Indeed the dipole moment fluctuates $1 / \sqrt{1,000,000}=0.1 \%$ due to this statistics. The strong-strong simulation gives an emittance growth rate $0.8 \times 10^{-9}$ without fluctuation. The weak-strong simulation gives the emittance growth rate $0.4 \times 10^{-9}$ for the fluctuation of $0.12 \%$ as shown in Figure 1. The emittance growth agrees within the factor 2 . This means the emittance growth in the strong-strong simulation is caused by the numerical noise of $0.1 \%$. Strong-strong simulation with less macro-particle do not have an ability for the prediction of luminosity decrements of $\sim 10^{-9}$ in 
the case of LHC.

Figure 2 shows the emittance growth given by the strongstrong simulation. Plot (a) shows an oscillation and growth of the emittance which indicates an excitation of a coherent motion. The growth rate is summarized in plot (b), where $\tau_{c o r}$ is the correlation time (turn) of the fluctuation ( $\tau_{c o r}=1$ is the default in this paper). The growth rate at $\delta x / \sigma=0.12 \%$ is comparable with that without fluctuation. The fluctuation is close to the numerical noise level of the macro-particle model. These results show that the fluctuation with $0.1 \%$ of the beam size is critical for one day luminosity life time. The emittance growth is somewhat higher than that given by the weak-strong simulation. Coherent motion may affect the growth. The growth for fluctuations with 100 turns correlation time is also plotted. The tolerance to fluctuations decreases with $\sqrt{\tau_{\text {cor }}}$.
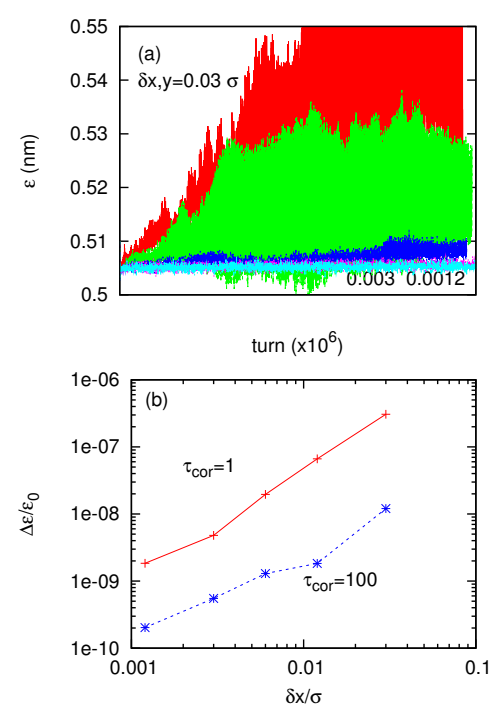

Figure 2: Emittance growth due to the fluctuation given by the strong-strong simulation. $t_{c o r}$ is the correlation time (in turns) of the fluctuation.

\section{Noise of the bunch by bunch feedback system}

A simulation has been performed for the second type of noise. An excitation of the beam-beam mode is the source of the emittance growth, therefore strong-strong simulation is essential for this study.

Figure 3 shows the emittance growth for the noise. Plot (a) depicts the evolution of emittance for various feedback gains with a kick noise $\delta x=0.02 \mu \mathrm{m}(0.12 \%$ of $\sigma)$. Emittance growth is seen, but coherent motion seems to be suppressed by the feedback system. The simulations were performed for several higher $\delta x$, and the growth rates are summarized in plot (b). The growth rates agree well with an analytical estimate [2].

Resolution of the position monitors also affect the beam fluctuation in Eq.(6). Emittance growth is slower at a higher gain for a given kicker noise, while it is faster for a given monitor resolution.
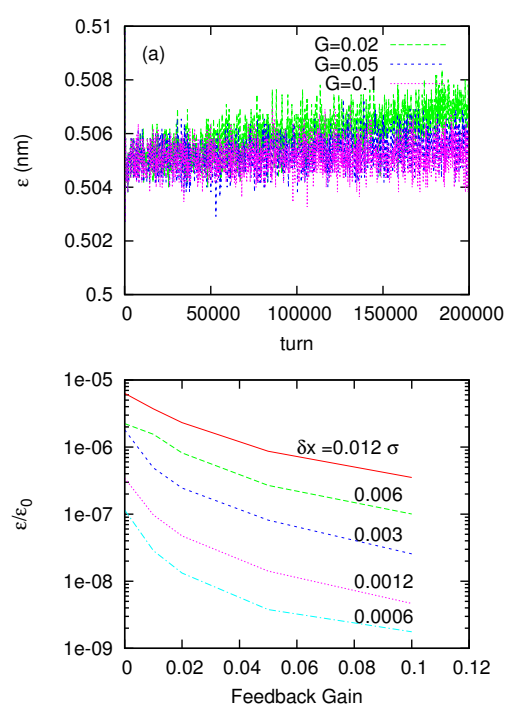

Figure 3: Evolutions of Dipole moment (a) and emittance (b) for various feedback gain with the kick noise $\delta x=0.02 \mu \mathrm{m} .(0.12 \%$ of $\sigma)$

\section{CONCLUSION}

Emittance growth and luminosity decrease due to external noise in a beam-beam collision system have been studied. To achieve a 1 day luminosity life time, the noise $\left(\delta x / \sigma_{x}\right)$ should be $0.1 \%$ for turn by turn noise $\left(t_{c o r}=1\right.$ turn). If the correlation time of the noise is 100 turns, the tolerance is $1 \%$. The tolerance roughly scales with correlation time as $\sqrt{t_{c o r}}$. The noise level of $0.1 \%$ correspond to phase fluctuation of $0.6 \mathrm{mrad}$ using $\Psi_{R F}=$ $10^{-3} \omega_{R F} \sigma_{x} / c \tan \phi$, where $\omega_{R F}=2 \pi \times 400 \mathrm{MHz}, \phi=$ $0.22 \mathrm{mrad}$.

For the transverse bunch by bunch feedback, the noise level, $\delta x_{k i c k} / \sigma=0.0006$ and $\mathrm{G}=0.1$, is about the limit of the luminosity decrement of $10^{-9}$. The corresponding monitor resolution is $\delta x_{\text {mon }}=0.0006 / G=0.006=$ $0.6 \%$, the resulting fluctuation in Eq. 6 is $0.1 \%$. For feedback system with lower gain, the monitor resolution is less stringent.

We acknowledge the support of the European Community-Research Infrastructure Activity under the FP6 "Structuring the European Research Area" programme (CARE, contract number RII3-CT-2003-506395)

\section{REFERENCES}

[1] T. Sen and J. A. Ellison, Phys. Rev. Lett., 77, 1051 (1996).

[2] Y.I. Alexahin, Nucl. Instru. and Methods in Phys. Res.,A 391, 73 (1996).

[3] K. Ohmi et al., in this proceedings (THPAN040). 